high leverage in order to provide resistant (stable) results in the presence of outliers and high leverage.

Results Based on 8949 employments records, dosimetric measurements of gamma radiation were significantly correlated with radon exposure $(r=0.499)$, duration of employment $(r=0.429)$, year of exposure $(r=0.239)$, and ore production $(r=0.230)$. Age was inversely related to gamma dose. Regression analysis showed that individual dosimetric readings can be modestly predicted by individual work history and geological characteristics of Ontario uranium mines $(\mathrm{p}<0.001, \mathrm{R} 2=0.374)$. Additional sources of variation are likely related to individual variability that could not be accounted for in this ecological assessment.

Conclusions Reconstructed gamma dose provides modest agreement with individual dosimetric readings.

\section{AN ASSESSMENT OF THE IMPACT OF MISCLASSIFICATION ERROR ON AN ESTIMATE OF EFFECT FOR OCCUPATIONAL EXPOSURE TO TCDD- CONTAMINATED CHEMICALS AND ISCHAEMIC HEART DISEASE}

Laura Scott. University of Minnesota School of Public Health, Minneapolis, MN, USA

\subsection{6/oemed-2014-102362.117}

Objectives To quantify the effect of exposure and disease misclassification on the odds ratio (OR) for ischaemic heart disease (IHD) mortality in a retrospective cohort study of trichlorophenol workers exposed to 2,3,7,8-tetrachlorodibenzo-p-dioxin (TCDD).

Method Exposure and disease classification parameters were defined using specificity and sensitivity values either reported in the peer-reviewed literature or calculated from available published data. The distribution shapes for each parameter were constructed based on expert judgment and were varied to address the lack of published information on the parameter distributions. Probabilistic uncertainty analysis, which uses Monte Carlo simulation techniques, was then used to sample each parameter distribution, calculating ORs corrected for outcome and exposure misclassification.

Results Correction for exposure and disease misclassification produced lognormal probability distributions of ORs for IHD with a wider range of possible values than calculated in the traditional epidemiological analysis. The median $\mathrm{OR}_{\text {corrected }}$ was larger than the $\mathrm{OR}_{\text {observed }}$ of 3.05 for all seven scenarios evaluated and ranged from 5.34 to 11.86 . Between $70 \%$ and $99 \%$ of the simulation trials yielded corrected ORs greater than the $\mathrm{OR}_{\text {observed }}$.

Conclusions The application of uncertainty analysis to a mortality study of workers occupationally exposed to TCDD-contaminated chemicals provides valuable insight into the magnitude and direction of misclassification error and the impact on an estimate of effect. Further refinement of the parameter distributions and adjustment of the $\mathrm{OR}_{\text {observed }}$ for other study limitations will be necessary to determine whether a true causal relationship between exposure and disease exists or if the effect observed in these workers is an artefact of systematic error.

\section{USING META-DATA FROM OCCUPATIONAL STUDIES TO INFORM HAZARD IDENTIFICATION AND CANCER CONTROL: THE IARC MONOGRAPHS AND BEYOND}

Neela Guha, Dana Loomis, Kurt Straif. IARC, LYON, France

10.1136/oemed-2014-102362.118
Objectives To present extensions to the International Agency for Research on Cancer (IARC) Monographs process, providing examples of application of meta-data to identifying carcinogenic hazard identifications and research gaps, and the potential use for guiding cancer control efforts. Qualitative and quantitative approaches will be contrasted.

Method The IARC evaluation process typically employs summary level meta-data, in the form of systematic reviews, and pooled- and meta-analyses.

Results IARC has heavily relied on published occupational epidemiological studies to identify specific carcinogens in the workplace and to form a scientific basis for the protection of workers worldwide. The evaluations of carcinogenic risk are made by international working groups of independent scientists and are qualitative in nature. Meta-analyses prepared for IARC working groups can complement the qualitative process and have been crucial in several instances; for example, in identifying sufficient evidence for lung and bladder cancer in painters and limited evidence for increased risk of bladder cancer among dry cleaners exposed to tetrachloroethylene and among professional drivers (bus, taxi, truck) with high exposure to outdoor air pollution. Since IARC does not provide recommendations for regulation or legislation, meta-relative risks can also be used to calculate attributable fractions to guide cancer control efforts, for agents in which a causal association is assumed and exposure prevalence has been well-characterised.

Conclusions The IARC Monographs Programme is an authoritative source for the identification of carcinogenic hazards in the environment. Applying meta-analyses to the IARC process can be a useful tool for informing hazard identification and providing guidance for cancer control efforts.

\section{ESTIMATING PERSONAL EXPOSURE TO TRAFFIC- RELATED AIR POLLUTANTS AMONG ON-ROAD WORKERS}

Kirsten Almberg, Jordan Hepler, Margaret Sietsema, Matthew Schmitz, Lorraine Conroy. University of Illinois at Chicago, Chicago, IL, USA

\subsection{6/oemed-2014-102362.119}

Objectives Many individuals in the U.S. work in close proximity to traffic-related air pollution. Often these jobs require physical activity which increases breathing rate and results in higher personal exposures to pollutants. The goal of this study was to systematically estimate and characterise personal exposure to traffic-related pollutants for those working on or near roadways. Method Concentrations of elemental carbon, carbon monoxide, and PM2.5 were measured along six traffic routes in the City of Chicago. These routes represent varying levels of exposure to traffic-related pollutants. Measurements were taken during peak and off-peak traffic hours during the summer and fall. All measurements were obtained from environmental monitoring equipment affixed to the back of a bicycle.

Results Air pollutant levels varied significantly across routes and time of day. Mean carbon monoxide levels ranged from 0.006$1.653 \mathrm{ppm}$ across the sampling events. The geometric mean elemental carbon level was $1.75 \mu \mathrm{g} / \mathrm{m}^{3}$ and ranged from 0.23 to $8.38 \mu \mathrm{g} / \mathrm{m}^{3}$. The geometric mean $\mathrm{PM}_{2.5}$ level was $39 \mu \mathrm{g} / \mathrm{m}^{3}$ and ranged from 16 to $270 \mu \mathrm{g} / \mathrm{m}^{3}$. Levels of elemental carbon and $\mathrm{PM}_{2.5}$ were significantly higher during peak traffic samples than off-peak traffic samples.

Conclusions This study uses novel methods to estimate exposure to those who are physically active along roadways including 
mobile. While there is considerable time and route variation in air pollutant measurement, the levels of air pollutants measured in this study may have serious health implications for those who perform physically demanding activities near or on roadways in the City of Chicago.

\section{QUANTIFYING HAZARDOUS OCCUPATIONAL EXPOSURES AND INJURIES IN HOME CARE: RESULTS FROM THE SAFE HOME CARE SURVEY}

${ }^{1}$ Margaret Quinn, 'Pia Markkanen, 'Catherine Galligan, 'Susan Sama, 'David Kriebel, ${ }^{1}$ Rebecca Gore, 'Laura Punnett, ${ }^{2}$ Letitia Davis, ${ }^{2}$ Angela Laramie, 'Natalie Brouillette, ${ }^{1}$ Daniel Okyere, ${ }^{1}$ Chuan Sun. ${ }^{1}$ University of Massachusetts Lowell, Lowell, MA, USA; ${ }^{2}$ Massachusetts Department of Public Health, Boston, MA, USA

10.1136/oemed-2014-102362.120

Objectives In countries with ageing populations, home care (HC) aides are among the fastest growing jobs. There are few quantitative studies of $\mathrm{HC}$ occupational safety and health (OSH) hazards. The objectives of this study were to quantify a range of $\mathrm{OSH}$ exposures and injuries among $\mathrm{HC}$ aides and to evaluate the variability across job categories.

Method HC aides were recruited for a survey via agencies that employ aides and schedule their visits with clients and through a labour union of aides employed directly by clients/families. The innovative questionnaire design included detailed questions about the most recent home visits, as well as about the individual aides' OSH experiences.

Results The final population included 1249 HC aides (634 agency-employed, 615 client-employed) contributing information on $3484 \mathrm{HC}$ visits. Among aides, in the past 12 months, 2\% had a needlestick or other sharps injury; $7 \%$ reported physical aggression and 19\% verbal aggression; $11 \%$ had a job-related injury resulting in lost work time or need for medical care; 33\% reported back pain: of these, $59 \%$ experienced pain once a week or more and $68 \%$ took medication for it. Among visits, in the past month, $62 \%$ involved patient handling while only $25 \%$ involved a patient handling device; $80 \%$ involved cleaning, $10 \%$ indoor cigarette smoke. Agency-employed versus client-employed aides differed by amount of client handling, use of sharps, and characteristics of clients.

Conclusions Aides experience substantial OSH exposures and injuries. Quantification of hazards is useful to prioritise resources for the development of preventive interventions and to provide a foundation for etiologic research.

\section{EVALUATION OF CUMULATIVE EXPOSURE TO CARBON BLACK AND LUNG CANCER RISK AMONG US CARBON BLACK WORKERS}

${ }^{1}$ Linda Dell, ${ }^{1}$ Alexa Gallagher, ${ }^{1}$ Lori Crawford, ${ }^{2}$ Rachael Jones, ${ }^{1}$ Kenneth Mundt. ${ }^{1}$ ENVIRON International Corp., Amherst, MA, USA; ${ }^{2}$ University of Illinois at Chicago, Chicago, IL, USA

\subsection{6/oemed-2014-102362.121}

Objectives To evaluate lung cancer mortality in relation to quantitative estimates of cumulative inhalable carbon black exposure among carbon black manufacturing workers.

Method Standardised mortality ratios (SMRs) were calculated for 5675 workers employed $\geq 1$ year since 1940 at 18 plants. Cox proportional hazards models were used to evaluate the association between cumulative inhalable carbon black exposure and lung cancer mortality risk for the "exposure" sub-cohort of 2099 men with complete work history records that allowed individual quantitative estimation of cumulative exposure.

Results All-cause $\mathrm{SMR}=0.80 \quad(95 \%$ confidence interval [CI] $=0.76-0.84)$, all-cancer $\mathrm{SMR}=0.80(95 \% \mathrm{CI}=0.73-0.88)$ and lung cancer mortality $\mathrm{SMR}=0.80(95 \% \mathrm{CI}=0.68-0.94)$ showed significant deficits. In the exposure sub-cohort, lung cancer mortality was not increased $(\mathrm{SMR}=0.68,95 \% \mathrm{CI}=0.44-1.00, \mathrm{n}=$ 20). Time-dependent Cox analyses of the exposure sub-cohort showed no positive associations: Hazards Ratio $[\mathrm{HR}]=0.20$ $(95 \% \mathrm{CI}=0.04-0.9)$ for 20 to $<50 \mathrm{mg} / \mathrm{m}^{3}$-years; $\mathrm{HR}=0.7(95 \%$ $\mathrm{CI}=0.20-2.0)$ for 50 to $<99 \mathrm{mg} / \mathrm{m}^{3}$-years; and $\mathrm{HR}=0.5(95 \%$ $\mathrm{CI}=0.1-1.7)$ for $\geq 100 \mathrm{mg} / \mathrm{m}^{3}$-years, compared with those with $<20 \mathrm{mg} / \mathrm{m}^{3}$-years.

Conclusions No excess lung cancer mortality or association between lung cancer mortality and time-dependent cumulative inhalable carbon black exposure were observed. However, few lung cancer deaths occurred among the exposure sub-cohort. Nevertheless, lung cancer mortality among the older, full cohort also was not increased using duration of employment as a surrogate of cumulative carbon black exposure.

\section{NEUROLOGIC AND REPRODUCTIVE EFFECTS OF SOLVENTS ON AUTOMOTIVE REPAIR WORKERS: ASSESSMENT OF EXPOSURE FOR THE BAY AREA SOLVENT STUDY (BASS)}

Katharine Hammond, Sa Liu, Sophie Horiuchi. University of California, Berkeley, Berkeley, CA, USA

\subsection{6/oemed-2014-102362.122}

Objectives To evaluate solvent exposures of automotive repair workers and investigate associated neurologic and reproductive effects.

Method Industrial hygienists conducted site-visits, air measurements, and interviews to prepare questions about task frequency, work practices, and products. Participants were recruited from IAMAW. Clinical exams evaluated peripheral neuropathy, neurocognition, colour discrimination, time to pregnancy, and urinary reproductive hormonal metabolites.

Results The 835 participants examined at the BASS clinic had worked over 3000 jobs at 1952 shops. Algorithms were developed to estimate individual solvent exposures in each year by integrating self-reported task frequency with MSDSs, measured and modelled airborne concentrations and dermal absorption. Major temporal changes occurred in source of exposures, e.g., solvent tanks accounted for $71 \%$ of solvent exposures in the 1960 s, $30 \%$ in $2000-04$, but only $7 \%$ in $2005-2012$; concomitantly, exposures from aerosol cans rose from $23 \%$ to $64 \%$ then $84 \%$, while composition changed dramatically, notably for hexane and perchloroethylene. Hexane was not used prior to 1989 and was eliminated from most products after 2000, when peripheral neuropathy was reported among automechanics. 52\% of the automechanics were exposed to hexane for a mean of 5.3 (0.1-12) years, and $80 \%$ of these had concomitant exposure to acetone, which potentiates neurologic effects of hexane in rodents. Cumulative hexane exposures were low: mean $=78.1$ $\mathrm{mg} / \mathrm{m}^{3}$ years $\left(\mathrm{TLV}=176 \mathrm{mg} / \mathrm{m}^{3}\right)$.

Conclusions Work histories were more complex than indicated in the pilot of 39 mechanics. Exposures were significantly affected by temporal trends in cleaning methods, composition of materials, and work practices. Epidemiologic studies must account for these changes. 